\title{
Italian pediatricians are largely against alternative weaning of the infant: a survey
}

\author{
Raffaella Panza ${ }^{1}$, Maria Elisabetta Baldassarre ${ }^{1 *}$, Valentina Rizzo ${ }^{1}$, Manuela Capozza $^{1}$ and Nicola Laforgia ${ }^{1}$
}

1. Department of Biomedical Science and Human Oncology, Neonatology and Neonatal Intensive Care Unit, “Aldo Moro” University of Bari, Bari 70100, Italy raffaella.panza@uniba.it; mariaelisabetta.baldassarre@uniba.it; rizzovale.vr@gmail.com; manuela.capozza@uniba.it; nicola.laforgia@uniba.it * Correspondence: Maria Elisabetta Baldassarre, M.D. Department of Biomedical Science and Human Oncology, Neonatology and Neona tal Intensive Care Unit, “Aldo Moro” University of Bari, Policlinico Hospital - Piazza Giulio Cesare n. 11 - 70124 - Bari, Italy. mariaelisabetta.baldassarre@uniba.it; cell +393296114818, fax +390805592991

\begin{abstract}
Background: Parents are increasingly fascinated by alternative weaning methods, such as baby-led or vegetarian weaning. However, international pediatric societies are still cautious towards alternative weaning methods, due to their significant risk of nutritional deficiencies. The aim of this study is to describe the attitude of Italian pediatricians towards unconventional weaning, with particular regard to vegetarian and baby-led. (2) Methods: A 20-question questionnaire was sent to Italian pediatricians, from January to December 2019; (3) Results: Responses were received from $73 / 1000(7.3 \%)$ pediatricians. The vast majority of surveyed pediatricians $(78.1 \%)$ is familiar with baby-led and vegetarian weaning, but only $24.7 \%$ is in favor of their practice. A significant number of pediatricians $(63.0 \%)$ received request from parents for an alternative weaning regimen. (4) Conclusions: The survey revealed a significant gap between pediatricians' attitude and parental demand concerning unconventional weaning. This could significantly impair the alliance between parents and pediatricians with the risk to expose infants and children to severe nutritional deficiencies due to self-management by parents with poor surveillance from health professionals.
\end{abstract}

Keywords: “Weaning"[Mesh], “Infant; newborn”[Mesh], “Diet; vegetarian”[Mesh], Baby-led weaning, Complementary feeding

\section{Introduction}

Weaning is the time period in which breast milk or infant-formula are gradually substituted by complementary foods. Traditionally, babies are first introduced to solid foods using spoon-feeding of suitable thin purées. Afterwards, according to infant's age and skills, foods gradually shift towards family foods[1].

Lately, the timing for introducing complementary foods and the methods of feeding have significantly changed: for instance, over the last 10 years vegetarian and vegan diets have become significantly popular, with a reported increase in prevalence of $350 \%$ [2]. To be more precise, vegetarianism is a multi-faceted regimen that can be further classified as follows:

- Semi-vegetarianism (includes birds and fishes);

- Lacto-ovo-vegetarians (includes dairy products and eggs);

- Lacto-vegetarians (includes dairy products);

- Veganism (total absence of any food of animal origin); 
- Macrobiotic diet (requires cooking the food as natural as possible, trying not to lose its properties; it is based on the Japanese philosophy of ying and yang; some meat or animal derivatives may be allowed)[3].

Over the last two decades also Baby-led weaning (BLW) has become particularly popular in the United Kingdom, New Zealand[4] and more recently in Europe[5], without a strong supporting evidence [6] and any mention in WHO's recommendation[7]. The term "baby-led weaning" was first coined by Gill Rapley in 2005[8] and indicates a method that allows the infant to lead the eating process by encouraging independence in choosing which food and the amount they will eat $[9,10]$. In this model, foods, preferably those consumed by the family and easy to handle and eat, are offered to the infant as finger foods, allowing the child to feed alone, promoting independence and an intense sensory exploration, unlike the traditional method, in which parents spoon-feed purées to their children (parent-led) and gradually adapt the food texture[11,12]. The key features of BLW are that, from six months of age, infants participate in family mealtimes and they feed themselves since the beginning of complementary feeding[13]. In this way the child himself controls the weaning process (hence the term "baby-led") and decides what, how much and how quickly to eat. The infant is an active partner in the feeding process, and not a passive recipient filled with food[8]. There is an increasing popularity of this method and BLW supporters suggest that it offers a range of benefits to babies, from better appetite control to a wider diet and better motor skill development, but BLW is not part of any official weaning guidelines, because of the lack of evidence for both efficacy and safety.

While parents are increasingly fascinated by alternative weaning methods [4,14], international pediatric societies are cautious towards both vegetarian and BLW weaning $[15,16]$, due to the significant risk of nutritional deficiencies they may cause as a consequence of the limited variety of foods with lack of medical supervision .

The aim of this study is to describe the attitude of Italian pediatricians towards unconventional weaning, with particular regard to vegetarian and baby-led weaning.

\section{Materials and Methods}

Italian pediatricians were surveyed by means of a 20 -question questionnaire that covered the following areas of interest:

\section{Personal beliefs on weaning;}

2. Knowledge and attitude towards baby-led and alternative weaning methods;

3. Appropriateness of alternative diets for weaning, in relation to the correct growth of the baby;

4. Precautions, feeding strategies and supplements applied to ensure proper development;

\section{Interrelation with parents.}

The questionnaire was submitted from January 2019 to December 2019 to one thousand Italian primary care pediatricians that were randomly selected from the mailing list of the Italian Federation of Pediatricians (Federazione Italiana Medici Pediatri, FIMP). An invitation email was sent out to the selected pediatricians to participate anonymously in a larger study assessing also weaning methods in ex-preterm infants [17] and prevalence of unconventional weaning regimens among their patients[14]. 
The questionnaire was produced on the basis of national and international guidelines provided by the most influencing pediatric societies (European Society for Pediatric Gastroenterology Hepatology and Nutrition - ESPGHAN; Academy of Nutrition and Dietetics -ADA, American Academy of Pediatrics - AAP, and Italian Pediatric Society SIP).

Data were uploaded as spreadsheet and analyzed using Microsoft Excel, version 16.30. Categorical variables were reported as proportions.

The participation was voluntary and written consent was not needed. Ethical approval was not sought since all data were anonymous.

\section{Results}

We received 73/1000 (7.3\%) questionnaires filled out by pediatricians from several Italian regions. Questionnaires were evaluated by two researchers (R.P. and M.C.). The main findings are reported below. (Tables 1-4)

Table 1. General attitude

\begin{tabular}{|c|c|}
\hline Item & Response, \% (n) \\
\hline \multicolumn{2}{|l|}{ Solid food introduction } \\
\hline - $\quad$ Before 4 months & $0(0)$ \\
\hline - $\quad$ Between 4 and 6 months & $83.6(61)$ \\
\hline - $\quad$ After 6 months & $16.4(12)$ \\
\hline \multicolumn{2}{|l|}{ Number of times recommended to feed after weaning } \\
\hline - $\quad$ Less than 2 & $6.8(5)$ \\
\hline - $\quad$ Between 2 and 4 & $24.7(18)$ \\
\hline - $\quad$ Between 4 and 6 & $52.1(38)$ \\
\hline - $\quad$ More than 6 & $0(0)$ \\
\hline - $\quad$ Based on child's request & $16.4(12)$ \\
\hline \multicolumn{2}{|l|}{ Breastfeeding duration } \\
\hline $\begin{array}{l}\text { - Up to the term set by the pediatrician in agreement with the } \\
\text { mother }\end{array}$ & $31.5(23)$ \\
\hline - Up to child's request & \\
\hline In favor of having the child at the table with parents & \\
\hline - Yes & $93.2(68)$ \\
\hline
\end{tabular}




\begin{tabular}{|l|l|}
\hline - No & $6.8(5)$ \\
\hline $\begin{array}{l}\text { In favor of satisfying child's dietary requests for foods deemed suitable } \\
\text { - Yes }\end{array}$ & \\
- No & $95.9(70)$ \\
& $4.1(3)$ \\
\hline
\end{tabular}

Pediatricians were surveyed on which foods they usually discourage. The most mentioned were cod, fish, and egg; but also tomato, strawberry, legumes, red meat, dried fruit, foods with gluten, fresh fruit, chocolate, foods with excess fiber, and salt were often discouraged.

Table 2. Attitude towards alternative weaning methods (baby-led and vegetarian weaning)

\begin{tabular}{|c|l|}
\hline \multicolumn{1}{|c|}{ Item } & Response, \% (n) \\
\hline $\begin{array}{c}\text { Knowledge of the practice of alternative weaning } \\
\text { - Yes }\end{array}$ & $78.1(57)$ \\
For or against alternative weaning methods? & $21.9(16)$ \\
- In favor & \\
- Against & $24.7(18)$ \\
\hline Willing to supervise an alternative weaning regimen for their patients & $75.3(55)$ \\
- Yes & $26.0(19)$ \\
\hline
\end{tabular}

As for the dietary regimen, $42.5 \%$ are in favor of the use of foods in which children show interest, whereas $31.5 \%$ are in favor of the use of foods chosen according to the parents' desires. One fourth of pediatricians $(26.0 \%)$ declined to respond.

Table 3. Appropriateness of alternative diet in relation to the correct growth of the infant

\begin{tabular}{|c|l|}
\hline \multicolumn{1}{|c|}{ Item } & Response, \% (n) \\
\hline Appropriateness to ensure correct weaning & \\
\hline
\end{tabular}




\begin{tabular}{|c|l|}
\hline - Yes & $8.2(6)$ \\
$\bullet \quad$ No & $91.8(67)$ \\
\hline Appropriateness to ensure proper growth & \\
$\bullet \quad$ Yes & $13.7(10)$ \\
- No & $86.3(63)$ \\
\hline
\end{tabular}

The sample was also surveyed on the strategies applied to ensure proper growth and development of their patients. Nearly two thirds of pediatricians $(68.5 \%)$ suggest monitoring critical micronutrients and treat deficiencies, whereas the remaining $31.5 \%$ prefers to prevent any deficiency by supplementing all children on an alternative.

When asked about the most critical micronutrients to monitor, pediatricians' responses were: Vitamin B12 86.3\% (63); Iron 82.2\% (60); Vitamin D 51.2\% (41); Calcium 42.5\% (31); Essential Amino-acids 41.1\% (30); Omega-3 27.4\% (20); Zinc 20.5\% (15).

The most recommended foods to increase protein intake in cases of children on alternative diets were: Legumes 80.8\% (59); Egg yolk 50.7\% (37); Cereals 45.2\% (33); Egg white $35.6 \%$ (26); Cheese 32.9\% (24); Cow's milk and derivatives 31.5\% (23); White Meat 28.8\% (21); Red meat $32.9 \%$ (24).

The most recommended supplements were: Vitamin D 56.2\% (41); Breast milk 49.3\% (36); Vitamin B12 Supplements 47.9\% (35); Iron Supplements 47.9\% (35); DHA 32.9\% (24); Iron-fortified Cereals 24.7\% (18); Vitamin A, C and zinc in lower percentages.

Table 5. Relationship between the pediatrician and parents

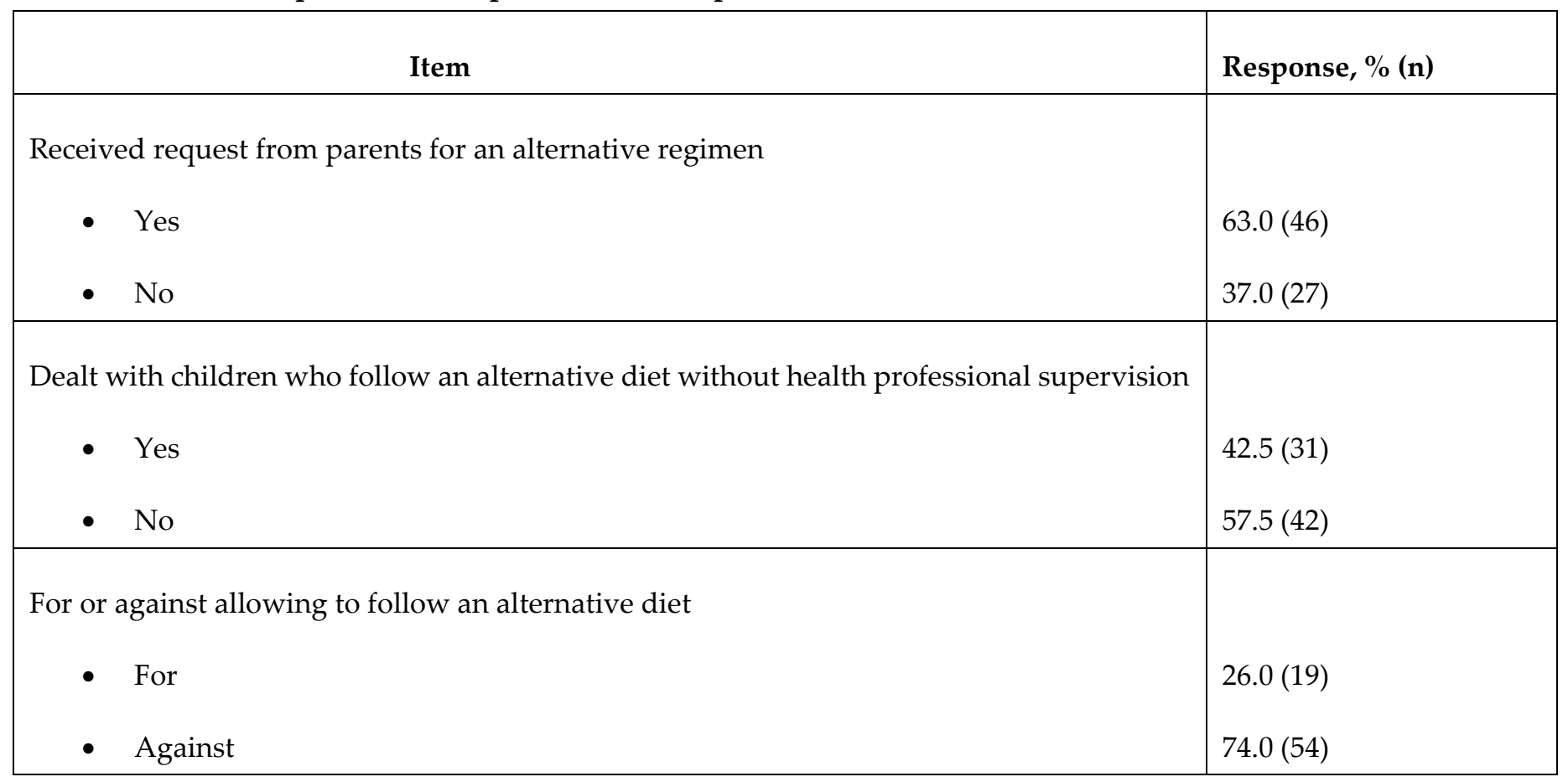




\section{Discussion}

The present survey showed that $78.1 \%$ of pediatricians who answered to the questionnaire is familiar with alternative weaning practices such as baby-led weaning (BLW) and vegetarian weaning, however only $24.7 \%$ is in favor of their practice. Apparently, it is increasingly common to find parents who require unconventional weaning: in our survey, the pediatricians who received request from parents for an alternative regimen were $63.0 \%$ of the whole sample, a significant and unexpected number. Nonetheless, the overall low response rate $(7.3 \%)$ may have biased this finding, since it could be argued that only pediatricians who are more sensitive to the topic due to parental requests accepted to respond.

Most of the interviewed pediatricians (91.8\%) thinks that alternative diets are not adequate to ensure the correct weaning of the infant and $86.3 \%$ of them believes that it is not even appropriate to guarantee the correct growth of the child.

A significant number of pediatricians (93.2\%) suggests having the child sit at the table with parents during meals, according to one of the basic principles of BLW.

Although BLW is not especially mentioned in WHO's recommendations[7], its popularity is constantly growing, since parents appreciate that infants experience the family diet and participate in family mealtimes[18].

It is very important that an adult closely supervise the food offered and the entire feeding process to prevent any risk of choking, for example by avoiding crisp hard vegetables and whole nuts[19].

Regarding vegetarian weaning, it is known that people following vegetarianism or veganism are gradually increasing, as well as the number of children on a vegetarian or vegan $\operatorname{diet}[2]$.

A recent review conducted on 360 Italian families showed that $8.6 \%$ of mothers were vegetarian or vegan and $9.2 \%$ of infants were weaned according to a vegetarian or vegan $\operatorname{diet}[14]$. In this review, Baldassarre et al showed that vegetarian/vegan mothers are more numerous than fathers ( $8.6 \%$ vs $2.8 \%)$ and closer to the percentage of infants weaned according to alternative feeding regimens (9.2\%). Maternal eating habits are more likely to influence the weaning of infants, as evidenced by the fact that among alternative weaned infants vegetarian/vegan mothers are $51.5 \%$ compared to $27.3 \%$ of fathers.

To date, there are no data to support safety and feasibility of alternative weaning methods. The absence of clear guidelines and the fact that many scientific societies[15,16] discourage alternative weaning methods because of the risk of nutritional deficiencies determine that the vast majority of pediatricians stands against novel practices such as BLW and vegetarian weaning[20]. It is well known that the smaller the variety of foods, the higher the risk of clinical or sub-clinical nutritional deficiencies in infants of vegetarian/vegan women or in children who follow alternative weaning methods[21,22].

The main drawback of this negative and erratic attitude of pediatricians towards alternative weaning regimens is the increasing number of families not considering their pediatrician as a reliable nutritional guide for this delicate process, with lack of supervision on child's health. 
Both BLW and vegetarian weaning, especially if self-managed without the supervision of the pediatrician, can cause severe nutritional deficiencies and complications, e.g. failure-to-thrive and possible serious health consequences[22]. An attitude of distrust towards the pediatrician is not uncommon from parents who choose alternative regimens, so in many cases families decide to follow an alternative diet without pediatric consultation[14].

The lack of nutritional knowledge of alternative diets and of pediatrician's control may be responsible of the increased risk of serious nutritional deficiencies for infants and young children undertaking alternative weaning regimens. It should be emphasized that alternative weaning methods are not suitable for ex preterm infants, a more vulnerable population $[23,24]$.

Our survey, regarding breastfeeding, shows that $68.5 \%$ pediatricians suggested continuing it according to child's request, whereas $31.5 \%$ set a fixed term in agreement with the mother.

Breast milk is the main caloric source for the newborn during alternative weaning, so maternal nutrition should include vitamin B12 supplementation and the right intake of vitamin $\mathrm{D}$, calcium and essential fatty acids.

In case of formula feeding during vegetarian weaning, rice or soy milk is able to provide the right amounts of vitamin B12, calcium, iron, zinc, iodine and DHA[15].

The questionnaire also investigated possible strategies and advice to be implemented if an alternative weaning or feeding regimen is undertaken and $68.5 \%$ pediatricians suggest monitoring micronutrients such as iron, vitamin B12, vitamin D and essential amino acids over time, in order to diagnose and treat possible deficiencies early. The remaining $31.5 \%$ prefer to give supplements to all children as a preventive measure. According to the present survey, the most recommended supplements were Vit. D (56.4\%), Vit. B12 $(47.9 \%)$, iron supplements (47.9\%), breast milk (49.3\%), and DHA $(32.9 \%)$.

To increase protein intake, the main choices were: legumes (80.8\%), egg yolk (50.7\%), and cereals $(45.2 \%)$.

In summary, our survey shows that Italian pediatricians follow some of the main criteria for a well-planned alternative diet in infancy[25], namely:

- Consume various plant foods;

- Limit the fiber intake;

- Choose vegetable fats favoring sources of omega-3 fatty acids and monounsaturated oils, and limiting trans-saturated fats and tropical oils;

- Consume calcium-rich foods (e.g. dairy products);

- Supplement vitamin D (1000-1200 IU/day if breastfed, or 600-800 IU/day if formula fed with vitamin D-enriched formula);

- Consume adequate amounts of vitamin B12 or supplement the lactating mother and the infant.

These criteria are meant to reduce the risk of nutritional deficiencies, especially of micronutrients, such as iron, zinc and vitamin B12. Since the high consumption of fiber and phytates inhibits the absorption of such micronutrients, their intake should be limited. In 
case of vegetarian/vegan weaning, meat should be replaced with protein-rich foods, such as legumes, soy and soy derivatives (milk, yogurt, tofu). Dried fruits or oilseeds should be added to baby food and yogurt (soy yogurt if vegan diet) to increase protein variety and caloric intake[14].

Another problem is the limited availability of commercial baby foods for vegetarian or vegan weaning. Moreover, as some commercial vegetarian/vegan foods contain animal-derived gelatin, meat, and fish oil, vegetarian parents often prefer to make their own baby foods, with the risk of exposing the child to unbalanced preparations, with high sodium content and reduced intake of energy, proteins, fats, iron and zinc.[26]. It is rather advisable to increase the consumption of soy products, dried beans, wheat germ, avocado, ground nuts, and nut butters, all of which are good sources of these nutrients.

To sum up, at the start of weaning, baby foods should be creamy, easy to chew, with ironand zinc-enriched cereals and no fibers in order to increase absorption. As far as flours are concerned, those enriched with iron and calcium (iron: $10 \mathrm{mg} / 100 \mathrm{~g}$; calcium: 400-560 $\mathrm{mg} / 100 \mathrm{~g}$ ) should be preferred to reduce the risk of iron-deficiency anemia and altered bone metabolism. To increase iron absorption it is useful to add few drops of lemon, as a source of vitamin $\mathrm{C}$.

The strength of our survey is to provide a general overview on the attitude of Italian pediatricians towards an emerging trending topic, i.e. alternative weaning regimens.

However, our results must be interpreted with caution due to the retrospective nature of this study and the low response rate, which may impair the soundness of our findings.

\section{Conclusions}

The survey revealed a significant gap between pediatricians attitude, not univocal and standardized, and parental demand regarding unconventional weaning with possible reduced capacity to cope with the needs and requests of families.. This leads to less satisfaction of many families concerning their relationship with the pediatrician and negative effects on the therapeutic alliance.

A prepared pediatrician, capable of providing the right answers to the requests of parents and explaining the pros and cons of the various methods, is absolutely needed to ensure the best growth and health of infants.

Alternative weaning should not be allowed as a parents' self-determination. Pediatricians have to guide highly motivated families following an alternative diet to provide all nutritional requirements. Furthermore, a careful follow-up of the infant is needed, considering that the greater the food restrictions, the greater the risk of deficit.

During breastfeeding and in general during childhood, as well as during pregnancy, correct information from an expert pediatrician or nutritionist is essential, to improve nutritional care, monitor any nutritional deficiencies and prescribe supplements if necessary.

Author Contributions: M.E.B and R.P. conceptualized the study and designed the questionnaire; they analyzed and interpreted data, and reviewed the initial manuscript. M.C., V.R. and R.P. wrote the first draft of the paper. N.L. and M.C. made substantial contributions to data analysis and interpretation. N.L. reviewed the initial manuscript. The final version of the manuscript was critically revised and finally approved as submitted by all the authors.

Funding: This research did not receive any specific grant from funding agencies of public, commercial, or not-for-profit sectors. Dr. Raffaella Panza received a fellowship funded by Mellin 
S.p.A. (Milan, Italy) to attend the Doctorate (PhD) course in Biomolecular Pharmaceutical and Medical Sciences of University of Bari - Aldo Moro.

Institutional Review Board Statement: Ethical review and approval were waived for this study, due to the fact that participation was voluntary and all data were anonymous.

Informed Consent Statement: Informed consent was waived since all data were anonymous.

Data Availability Statement: The data presented in this study are available in the article.

Acknowledgments: We acknowledge the No-Profit Association "La Medicina in uno Scatto" for the support in conducting the present survey.

Conflicts of Interest: None of the authors has any competing interests relevant to this article.

\section{References}

1. Castenmiller, J.; de Henauw, S.; Hirsch-Ernst, K.I.; Kearney, J.; Knutsen, H.K.; Maciuk, A.; Mangelsdorf, I.; McArdle, H.J.; Naska, A.; Pelaez, C.; et al. Appropriate age range for introduction of complementary feeding into an infant's diet. EFSA J. 2019, 17, doi:10.2903/j.efsa.2019.5780.

2. Ferrara, P.; Corsello, G.; Quattrocchi, E.; Dell'Aquila, L.; Ehrich, J.; Giardino, I.; Pettoello-Mantovani, M. Caring for Infants and Children Following Alternative Dietary Patterns. J. Pediatr. 2017, 187, 339-340.e1, doi:10.1016/j.jpeds.2017.04.053.

3. Agnoli, C.; Baroni, L.; Bertini, I.; Ciappellano, S.; Fabbri, A.; Papa, M.; Pellegrini, N.; Sbarbati, R.; Scarino, M.L.; Siani, V.; et al. Position paper on vegetarian diets from the working group of the Italian Society of Human Nutrition. Nutr. Metab. Cardiovasc. Dis. 2017, 27, 1037-1052, doi:10.1016/j.numecd.2017.10.020.

4. Cameron, S.L.; Taylor, R.W.; Heath, A.L.M. Parent-led or baby-led? Associations between complementary feeding practices and health-related behaviours in a survey of New Zealand families. BMJ Open 2013, 3.

5. Caroli, M.; Mele, R.M.; Tomaselli, M.A.; Cammisa, M.; Longo, F.; Attolini, E. Complementary feeding patterns in Europe with a special focus on Italy. Nutr. Metab. Cardiovasc. Dis. 2012, 22, 813-818.

6. Brown, A.; Jones, S.W.; Rowan, H. Baby-Led Weaning: The Evidence to Date. Curr. Nutr. Rep. 2017, 6, $148-156$.

7. Global strategy for infant and young child feeding. World Heal. Organ. UNICEF 2003.

8. Rapley, G. Baby-led weaning: transitioning to solid foods at the baby's own pace. Community Pract. 2011, 84, $20-23$.

9. Cichero, J.A.Y. Introducing solid foods using baby-led weaning vs. spoon-feeding: A focus on oral development, nutrient intake and quality of research to bring balance to the debate. Nutr. Bull. 2016, 41, 72-77, doi:10.1111/nbu.12191.

10. Rapley, G.; Forste, R.; Cameron, S.; Brown, A.; Wright, C. Baby-Led Weaning: A New Frontier? Infant, Child, Adolesc. Nutr. 2015, 7, 77-85, doi:10.1177/1941406415575931.

11. Cameron, S.L.; Heath, A.L.M.; Taylor, R.W. How feasible is Baby-Led Weaning as an approach to infant feeding? A review of the evidence. Nutrients 2012, 4, 1575-1609.

12. Pesch, M.; Shubeck, A.; Burrows, H. Baby-led weaning: introducing complementary foods in infancy. Contemp. PEDS J. 2019, 36, 24-8.

13. Rapley, G. Baby-led weaning: The theory and evidence behind the approach. J. Heal. Visit. 2015, 3, 144-151, doi:10.12968/johv.2015.3.3.144.

14. Baldassarre, M.E.; Panza, R.; Farella, I.; Posa, D.; Capozza, M.; Di Mauro, A.; Laforgia, N. Vegetarian and vegan weaning of the infant: How common and how evidence-based? a population-based survey and narrative review. Int. J. Environ. Res. Public Health 2020, 17, 1-17, doi:10.3390/ijerph17134835.

15. Lemale, J.; Mas, E.; Jung, C.; Bellaiche, M.; Tounian, P. Vegan diet in children and adolescents. Recommendations from the French-speaking Pediatric Hepatology, Gastroenterology and Nutrition Group (GFHGNP). Arch. Pediatr. 2019, 26, 442-450, doi:10.1016/j.arcped.2019.09.001.

16. Fewtrell, M.; Bronsky, J.; Campoy, C.; Domellöf, M.; Embleton, N.; Mis, N.F.; Hojsak, I.; Hulst, J.M.; Indrio, F.; Lapillonne, 
A.; et al. Complementary feeding: A position paper by the European Society for Paediatric Gastroenterology, Hepatology, and Nutrition (ESPGHAN) committee on nutrition. J. Pediatr. Gastroenterol. Nutr. 2017, 64, 119-132, doi:10.1097/MPG.0000000000001454.

17. Baldassarre, M.E.; Di Mauro, A.; Pedico, A.; Rizzo, V.; Capozza, M.; Meneghin, F.; Lista, G.; Laforgia, N.; Italian Society of Pediatrics (SIP), Italian Society of Neonatology (SIN), Italian Society of Pediatric Gastroenterology, Hepatology, and Nutrition (SIGENP) and Italian Federation of Paediatricians (FIMP) Weaning Time in Preterm Infants: An Audit of Italian Primary Care Paediatricians. Nutrients 2018, 10, doi:10.3390/nu10050616.

18. Rowan, H.; Harris, C. Baby-led weaning and the family diet. A pilot study. Appetite 2012, 58, 1046-1049, doi:10.1016/j.appet.2012.01.033.

19. Utami, A.F.; Wanda, D.; Hayati, H.; Fowler, C. "Becoming an independent feeder": infant's transition in solid food introduction through baby-led weaning. BMC Proc. 2020, 14, 18, doi:10.1186/s12919-020-00198-w.

20. Bettinelli, M.E.; Bezze, E.; Morasca, L.; Plevani, L.; Sorrentino, G.; Morniroli, D.; Giannì, M.L.; Mosca, F. Knowledge of health professionals regarding vegetarian diets from pregnancy to adolescence: An observational study. Nutrients 2019, 11, 1149, doi:10.3390/nu11051149.

21. Lemoine, A.; Giabicani, E.; Lockhart, V.; Grimprel, E.; Tounian, P. Case report of nutritional rickets in an infant following a vegan diet. Arch. Pediatr. 2020, 8-11, doi:10.1016/j.arcped.2020.03.008.

22. Farella, I.; Panza, R.; Baldassarre, M.E. The Difficult Alliance between Vegan Parents and Pediatrician: A Case Report. Int. J. Environ. Res. Public Health 2020, 17, 6380, doi:10.3390/ijerph17176380.

23. Baldassarre, M.E.; Di Mauro, A.; Pedico, A.; Rizzo, V.; Capozza, M.; Meneghin, F.; Lista, G.; Laforgia, N. Weaning time in preterm infants: An audit of italian primary care paediatricians. Nutrients 2018, 10, 616, doi:10.3390/nu10050616.

24. Baldassarre, M.E.; Giannì, M.L.; Di Mauro, A.; Mosca, F.; Laforgia, N. Complementary feeding in preterm infants: Where do we stand? Nutrients 2020, 12, 1259.

25. Baroni, L.; Goggi, S.; Battaglino, R.; Berveglieri, M.; Fasan, I.; Filippin, D.; Griffith, P.; Rizzo, G.; Tomasini, C.; Tosatti, M.A.; et al. Vegan nutrition for mothers and children: Practical tools for healthcare providers. Nutrients 2019, 11, 5, doi:10.3390/nu11010005.

26. Stordy, B.; Redfern, A.; Morgan, J.B. Healthy eating for infants-mothers'actions. Acta Paediatr. 1995, 84, 733-741, doi:10.1111/j.1651-2227.1995.tb13746.x. 